\title{
Marketing digital 4.0 para fortalecer el posicionamiento en el mercado manufacturero
}

Digital marketing 4.0 to strengthen positioning in the manufacturing market

\begin{tabular}{|c|c|}
\hline Ronald Aguilar & Juan Alvarado \\
\hline $\begin{array}{l}\text { ronalddm2207@gmail.com } \\
\text { ORCID: 0000-0001-9293-5089 }\end{array}$ & $\begin{array}{l}\text { jcam3010@hotmail.com } \\
\text { ORCID: 0000-0001-9018-8470 }\end{array}$ \\
\hline Universidad de Carabobo, Venezuela & Universidad de Carabobo, Venezuela \\
\hline
\end{tabular}

Artículo recibido en enero 2020 / Arbitrado en febrero 2020 / Publicado en mayo 2020

\section{RESUMEN}

Palabras clave:
El objetivo de esta investigación fue realizar un plan estratégico basado en el marketing 4.0 con el fin de fortalecer el mercado manufacturero de la empresa INRUE C.A ubicada en Valencia, estado Carabobo, Venezuela, dedicada a la fabricación, venta y distribución de ruedas industriales en hierro. Para ello, el estudio se apoyó en una investigación de campo y documental y como unidades de estudio se tomó una muestra representada por 22 clientes de los distintos Municipios del Estado Carabobo, a las que se aplicó un cuestionario tipo Escala de Likert. Los datos recolectados permitieron determinar la información necesaria para recomendar a la empresa sobre el diseño e implementación de un plan estratégico de Marketing 4.0 que permita posicionar la compañía en el mercado manufacturero.

Marketing digital 4.0, manufactura, mercado
ABSTRACT

Keywords:
The objective of this research was to carry out a strategic plan based on marketing 4.0 in order to strengthen the manufacturing market of the INRUE CA company located in Valencia, Carabobo state, Venezuela, dedicated to the manufacture, sale and distribution of industrial iron wheels. For this, the study was supported by a field and documentary research and as study units a sample represented by 22 clients from the different Municipalities of the Carabobo State was taken, to which a Likert Scale questionnaire was applied. The data collected made it possible to determine the information necessary to recommend to the company on the design and implementation of a strategic Marketing 4.0 plan that allows the company to position itself in the manufacturing market.

Digital Marketing 4.0, manufacturing, market 
INTRODUCCIÓN

Hoy en día, el proceso de digitalización rápida es un fenómeno del siglo XXI, ya que cada ámbito de la vida cotidiana y empresarial es total o parcialmente integrado con Internet. Por lo tanto, es crucial para cualquier empresa establecer su presencia en línea para atender la demanda de un nuevo lugar, atraer a los clientes a través de los canales de comercialización, para aprovechar al máximo las oportunidades en línea.

En términos generales, el marketing digital es una combinación de nuevas tecnologías que capta la atención de un público objetivo en un entorno de rápido desarrollo. Así bien, los sitios web, boletines y ofertas de correo electrónico, medios de comunicación social, en línea revistas y directorios, blogs, son todos ejemplos de canales en línea disponibles a las empresas. Una combinación de la identidad del contenido relevante para una determinada audiencia y un conjunto óptimo de canales para distribuir ese contenido, permite aumentar el conocimiento de la marca, clientes potenciales y el fortalecimiento de la lealtad y la satisfacción de la base de clientes emocionante. Esto lleva a la necesidad de tener una estrategia de valor añadido que tiene como objetivo aumentar las medidas de ayuda de las mejores técnicas de marketing online. .

Así mismo, la competitividad, como concepto, tiene una importante y decisiva connotación económica; de hecho, la competitividad de una economía nacional está construida sobre la de las empresas que operan en ella. Así, como afirma Concha, M. (2008), "... La habilidad de las empresas para obtener beneficios y mantener $\circ$ ganar participación en los mercados nacionales e internacionales genera una ventaja competitiva." (p.12).

En este contexto, cabe señalar la postura de Cobra, M. (2007), para quien "los rápidos cambios en los gustos del consumidor, en la tecnología y en la competencia, hacen que las organizaciones a nivel mundial traten de desarrollar estrategias para incorporar el mejoramiento continuo de los productos y servicios" (p. 254), ya que hoy día un buen producto y un buen precio ya no son suficientes ante la satisfacción de las cambiantes necesidades y deseos del consumidor, por lo que cada día se observan nuevas formas de mercadear productos y servicios, intensificándose así la competencia en los mercados.

De allí, que se planteó como objetivo de esta investigación, realizar un plan estratégico basado en el marketing 4.0 con el fin de fortalecer el mercado manufacturero de la empresa INRUE C.A ubicada en Valencia, estado Carabobo, dedicada a la fabricación, venta y distribución de ruedas industriales en hierro, poliuretano, goma, aluminio, carruchas, soportes livianos y pesados, entre otros productos.

\section{MATERIALES Y METODOS}

La presente investigación, descriptiva y de campo, utilizando como técnicas de recolección de datos la entrevista realizada al propietario de la empresa y la encuesta 
aplicada a una muestra de 22 clientes habitantes del Estado Carabobo, quienes respondieron acerca de la temática en estudio. La aplicación de estos instrumentos permitió identificar los posibles elementos que deben ser contemplados en el plan estratégico basado en el marketing digital 4.0 para fortalecer el posicionamiento del mercado manufacturero de la empresa INRUE C.A.

\section{RESULTADOS Y DISCUSIÓN}

Cuadro 1. Resultados del Guion de Entrevista

ITEM GERENTE GENERAL INRUE C.A.

1.- ¿En qué año la empresa INRUE C.A comenzó a funcionar?

2.- ¿Qué tipo de productos de manufactura elaboran en la empresa, cuentan con tecnología de vanguardia en la elaboración de sus productos?

3.- ¿Cuál es el perfil de los clientes que adquieren sus productos, las compras las realizan al mayor o al detal y cuenta actualmente con una cartera de clientes solida?

4.- ¿Cuáles son los factores que usted considera importantes para el éxito en una empresa de manufactura de este tipo, cuál es su competencia más fuerte y cercana, cuenta con estrategia de venta?
La empresa empieza a funcionar en el año 2009 con el nombre de INRUECA. La empresa INRUECA tenía desempeñándose antes de tener el nombre que hoy en día tenemos como Fundición Guyana Repuestos; para el año 2000 se disuelve la sociedad y allí pasa a llamarse INRUE C.A

Se elaboran productos a nivel ferretero, se fabrican y distribuyen ruedas industriales en hierro, poliuretano, goma, aluminio, andamios, carruchas, soportes livianos y pesados y revestimientos de rodillos y todo tipo de piezas especiales en poliuretano, en cuanto a tecnología la empresa posee maquinaria especializada para la elaboración de las piezas, aunque no es la más novedosa y actualizada del momento, pero fabrica con calidad sus productos. Las maquinarias de vanguardia son más costosas y se necesitan de una inversión muy amplia para la compra de dicha maquinaria

Nuestros clientes son distribuidoras a nivel ferretero, todos mis clientes me hacen compras al mayor, aunque muy pocas veces hay clientes que son recomendados y buscan que le fabrique una pieza en específico, y bueno gracias a Dios contamos con una cartera de clientes solida ya que les gusta la calidad de nuestras piezas.

Bueno yo considero el factor más importante para que la empresa se mantenga funcionando es la adquisición de la materia prima. También que la economía tenga una estabilidad en los precios, en cuanto a nuestra competencia más fuerte es Metalarte C.A que está ubicada en Maracaibo y Metalma C.A que está ubicada aquí mismo en Valencia de resto no tenemos competencia y mis estrategias de venta manejo actualmente nuestra cartera de clientes a los que les distribuimos nuestros productos, pero distribuidora propia no tenemos. 
5.- ¿Cuál es su estrategia para captar nuevos clientes, tiene acciones a tomar para estar por encima de su competencia, usted como gerente general y dueño de la empresa que ofrece que su competencia más cercana no ofrece?

6.- ¿Qué estrategias de marketing posee actualmente la empresa, considera usted que son las fallas que posee actualmente la empresa que evitan su posicionamiento en el mercado?
Bueno estrategias nuevas como tal no tenemos, nos llegan personas recomendadas por nuestra cartera de clientes que llegan nos compran los productos, lo más novedoso así que tenemos desde el año 2012 es nuestra cuenta en Facebook la cual es inrueca@hotmail.com y mi correo electrónico personal que es angelhpd@hotmail.com las acciones para estar por encima de mi competencia es que yo contribuyo con hacer bien mis piezas y productos para que cumpla con las necesidades del cliente y yo como Gerente General ofrezco calidad y eficiencia en la fabricación de nuestros productos porque también somos una empresa con larga trayectoria en el mercado ferretero y eso nos hace una empresa confiable.

Como les dije actualmente solo tenemos Facebook más allá del marketing, no poseemos más nada, nos gustaría abordar más en este tema. Nuestra principal falla que evita un crecimiento de la empresa en sus ventas y el posicionamiento es que no tenemos distribuidora, solo tenemos clientes a lo que les distribuimos nuestros productos pero me gustaría crear un espacio propio para que promocionemos y comercialicemos nuestros productos.

Fuentes: Aguilar, R., Alvarado, J. (2019)

De la entrevista realizada en la empresa, se puede inferir que la compañía tiene más de 8 años en el mercado manufacturero y sus clientes son fieles pero a su vez tienen debilidades en cuanto a la promoción y publicidad de sus productos así como la ubicación de su compañía no es la más adecuada para sus clientes, la empresa desea incursionar en el mercado ferretero de menudeo y aperturar una tienda en un lugar más cercano para su clientela, por este motivo se les propuso mediante un análisis DOFA crear un plan estratégico de Marketing con la finalidad de incrementar su posición en el mercado manufacturero de la región central del país específicamente en Carabobo.

Se realizó el análisis de la situación y a su vez identificaron los competidores existentes en cuanto a producto, precio, descuentos, ubicación, canales de distribución, políticas de venta, publicidad y promoción. Por otra parte, se estudió el entorno y situación del mercado, situación política, económica, legal, tecnológica, así como también el comportamiento del consumidor, costumbres del mercado y la situación de la empresa respecto a políticas de productos, finanzas, capacidad, personal y medios para finalmente realizar un análisis DOFA. 
En este caso, se analizó la situación arrojando como resultado que los competidores directos solo distribuyen a clientes específicos a nivel de mayor y no ofrecen variedad de productos, los precios son similares a los ofertados en la compañía y manejan las mismas políticas de ventas y canales de distribución. En cuanto a la empresa INRUE C.A se utilizó una herramienta de fácil uso como lo es el análisis DOFA que mide las Debilidades, Oportunidades, Fortalezas y Amenazas de la estrategia de marketing digital utilizada por la compañía, así como también su ubicación en el mercado. Las Debilidades y Fortalezas corresponden al interior, son propias del elemento a analizar y las Amenazas y Oportunidades muestran el entorno.

\section{Análisis DOFA}

\section{Debilidades:}

- No existen gran cantidad de visitas a la red social Facebook de la empresa y mantiene su mismo número de visitas mensuales ya que los clientes no tienen conocimiento de la existencia de la misma.

- Las actualizaciones de los productos promocionados por dicha red social no se realizan de manera continua sino esporádica.

- El posicionamiento quizás no esté bien dirigido al cliente que se quiere abordar.

- No existen ofertas innovadoras de productos nuevos.
- No existe posicionamiento alguno en redes.

- No hay publicidad de ningún tipo en ningún medio.

- La ubicación de la sede principal se encuentra alejada de la ciudad y los clientes deben trasladarse a largas distancias para adquirir su producto.

- No cuenta con un asesor especializado en el área para ayudar al cliente.

- No posee tienda de comercialización para ventas al público en general.

\section{Oportunidades:}

- No posee mayor competencia en el mercado, convirtiéndose esto en una gran ventaja ya que puede posicionarse en el ramo manufacturero de ruedas industriales como el número uno en calidad, senvicio y costo.

- Cada vez es mayor el crecimiento y penetración de la red y servicios de internet en el país y el mundo.

- Los costos de implementar estrategias de marketing digital son menores a los de cualquier plan de medios tradicional.

- Existen cada vez herramientas gratuitas al alcance de cualquier usuario o empresa para utilizar y promocionarse en la red.

- Los sitios pueden combinar estrategias de publicidad y de venta on line y de información para los usuarios, de este modo comparten y dividen los gastos e ingresos por publicidad. 
- Existen pequeñas campañas de publicidad que pueden hacerse de manera gratuita pero con gran dedicación temporaria.

- La web nos permite a diferencia de una ubicación local geográfica real, poder llegar a lugares inesperados donde alguien puede requerir nuestro producto o servicio.

- Existen herramientas que permiten el pago y compra en línea, con gran confianza permitiendo a las empresas vender y recibir pagos al instante.

- Con un buen sitio, un sitio atractivo y funcional, se puede complementar el espacio físico.

- Las comunicaciones y las tecnologías traen consigo mas y nuevas tendencias y oportunidades de negocio que pueden ser aprovechadas por las empresas.

- Los clientes están sugiriendo apertura de una nueva sede más céntrica en la ciudad trayendo consigo un incremento significativo en las ventas.

\section{Fortalezas:}

- Los clientes son fieles a los productos y canal de distribución ya que en su mayoría tienen en promedio más de cuatro años de antigüedad.

- Los productos ofrecidos son de excelente calidad.

- Los clientes adquieren los productos mensualmente garantizando así el ingreso para la empresa.
- Son de alta competencia en el ramo ferretero ya que sus productos son de excelente calidad a bajos costos.

- Los clientes recomiendan sus productos ampliando así levemente la cartera de los mismos.

\section{Amenazas:}

- En la web, así como aumentan las visitas y la promoción de nuevos modelos, también, existe desconfianza o violaciones a seguridad.

- Algunos contenidos de la web, pueden llegar a ser corrosivos si se utilizan para perjudicar una marca o una empresa.

- La web como bien se dice, es como una gran nube y mercado donde hay muchísimos oferentes y muchísimos demandantes, así que es un reto destacarse y ser queridos y recordados por los usuarios.

- Los datos y la protección de los mismos, puede estar en riesgo aunque surgen a diario nuevas maneras de protección.

\section{Presentación y análisis de resultados de la encuesta.}

A continuación se presentan los resultados estadísticos del instrumento aplicado a una muestra de la población, en este caso clientes de la empresa INRUE C.A ubicada en el Estado Carabobo, quienes respondieron de manera individual un cuestionario acerca de la temática que se desea conocer. 
Cuadro $\mathbf{N}^{\circ}$ 2. Antigüedad de cliente de INRUE, C.A.

\begin{tabular}{ccc}
\hline Opciones & Frecuencia & Porcentajes \\
\hline De $\mathbf{3}$ a $\mathbf{6}$ meses & 4 & $18 \%$ \\
De $\mathbf{6}$ meses a $\mathbf{1}$ año & 5 & $23 \%$ \\
De $\mathbf{1}$ año a $\mathbf{5}$ años & 7 & $32 \%$ \\
Más de $\mathbf{5}$ años & 6 & $27 \%$ \\
Total & 22 & $100 \%$ \\
\hline
\end{tabular}

Fuentes: Aguilar, R., Alvarado, J. (2019)

De estas respuestas debe inferirse que en su mayoría son clientes de más de cinco años de antigüedad y que el aumento de clientes en los últimos años no ha sido considerable.

Cuadro $\mathbf{N}^{\circ}$ 3. Le gusta la calidad de sus productos

\begin{tabular}{ccc}
\hline Opciones & Frecuencia & Porcentajes \\
\hline Si & 22 & $100 \%$ \\
No & 0 & $0 \%$ \\
Total & 22 & $100 \%$ \\
\hline
\end{tabular}

Fuentes: Aguilar, R., Alvarado, J. (2019)

Basándose en estas respuestas puede interpretarse que los clientes se encuentran satisfechos con la calidad de los productos que ofrece la empresa, así como también la satisfacción que produce en sus clientes y cubren todas sus necesidades y demandas.

Cuadro $\mathbf{N}^{\circ}$ 4. Con que frecuencia compra los productos de INRUE, C.A.

\begin{tabular}{ccc}
\hline Opciones & Frecuencia & Porcentajes \\
\hline Semanal & 1 & $5 \%$ \\
Quincenal & 4 & $18 \%$ \\
Mensual & 13 & $59 \%$ \\
Otros & 4 & $18 \%$ \\
Total & 22 & $100 \%$ \\
\hline
\end{tabular}

Fuentes: Aguilar, R., Alvarado, J. (2019)

Ante esta situación se observa el alto flujo de ventas que realiza la empresa en cortos intervalos de tiempo, es decir un mes o menos, desarrollando alto volumen de productos y alta demanda por satisfacer a sus clientes. 
Cuadro $\mathbf{N}^{\circ}$ 5. Cómo valora la calidad y elaboración de los productos de INRUE, C.A.

\begin{tabular}{ccc}
\hline Opciones & Frecuencia & Porcentajes \\
\hline Excelente & 11 & $50 \%$ \\
Buena & 9 & $41 \%$ \\
Normal & 2 & $9 \%$ \\
Regular & 0 & $0 \%$ \\
Total & 22 & $100 \%$ \\
\hline
\end{tabular}

Fuentes: Aguilar, R., Alvarado, J. (2019)

Esta información es bastante alentadora debido a que los productos que se elaboran dentro de la empresa son considerados de muy buena calidad por sus clientes y con ello lo hace un punto muy sólido para impulsar los productos de la misma ya que son basados en conceptos de gran calidad por los usuarios o clientes de hace muchos años

Cuadro $\mathbf{N}^{\circ}$ 6. Qué le parece el precio de sus productos

\begin{tabular}{ccc}
\hline Opciones & Frecuencia & Porcentajes \\
\hline Económicos & 12 & $54 \%$ \\
Muy económicos & 5 & $23 \%$ \\
Costosos & 5 & $23 \%$ \\
Regular & 0 & $0 \%$ \\
Total & 22 & $100 \%$ \\
\hline
\end{tabular}

Fuentes: Aguilar, R., Alvarado, J. (2019)

El gráfico anterior muestra que un 54\% respondió que están muy económicos, lo cual certifica en su mayoría que los productos son económicos y de fácil acceso monetario, dándole otra oportunidad de impulso ya que son productos de buena calidad y de fácil obtención económica.

Cuadro $\mathbf{N}^{\circ}$ 7. Le gustaría que se incluyeran nuevos productos en la empresa INRUE, C.A.

\begin{tabular}{ccc}
\hline Opciones & Frecuencia & Porcentajes \\
\hline Si & 20 & $91 \%$ \\
No & 2 & $9 \%$ \\
Total & 22 & $100 \%$ \\
\hline
\end{tabular}

Fuentes: Aguilar, R., Alvarado, J. (2019) 
En este punto se puede visualizar que existe un alto nivel de necesidad entre los clientes sobre innovar sus productos de acuerdo a la necesidad actual del mercado y las novedades que este va exigiendo en los crecimientos empresariales.

Cuadro $\mathbf{N}^{\circ}$ 8. A través de que medio se enteró de INRUE, C.A.

\begin{tabular}{ccc}
\hline Opciones & Frecuencia & Porcentajes \\
\hline Amigo/Cliente & 17 & $77 \%$ \\
Cerca del establecimiento & 3 & $14 \%$ \\
Redes Sociales & 2 & $9 \%$ \\
Otros & 0 & $0 \%$ \\
Total & 22 & $100 \%$ \\
\hline
\end{tabular}

Fuentes: Aguilar, R., Alvarado, J. (2019)

Aquí se evidencia la necesidad de impulsar y dar a conocer la empresa a través de medios publicitarios, y en nuestro estudio a través del marketing digital 4.0.

Cuadro $\mathbf{N}^{\circ}$ 9. Considera usted que la empresa INRUE, C.A., necesita más medios y redes sociales para su publicidad

\begin{tabular}{ccc}
\hline Opciones & Frecuencia & Porcentajes \\
\hline Si & 21 & $95 \%$ \\
No & 1 & $5 \%$ \\
Total & 22 & $100 \%$ \\
\hline
\end{tabular}

Fuentes: Aguilar, R., Alvarado, J. (2019)

Aquí se evidencia netamente la necesidad de tener un plan de marketing digital para dar a conocer e impulsar la empresa para lograr mayor captación de clientes, así como también dar a conocer sus productos e interactuar con los clientes para conocer sus necesidades.

Cuadro $\mathbf{N}^{\circ}$ 10. Le gustaría información de promoción y descuento de la empresa INRUE, C.A.

\begin{tabular}{ccc}
\hline Opciones & Frecuencia & Porcentajes \\
\hline Si & 18 & $82 \%$ \\
No & 4 & $18 \%$ \\
Total & 22 & $100 \%$ \\
\hline
\end{tabular}

Fuentes: Aguilar, R., Alvarado, J. (2019) 
Es importante observar este punto ya que los clientes sienten la necesidad de mantenerse informado sobre los productos que ofrecen, así como las posibles promociones que pueden desarrollarse dentro de la empresa observándose la necesidad del mercado y de cómo se cubrirá a través de un plan de marketing digital acorde al mercado actual

Cuadro $\mathbf{N}^{\circ}$ 11. Cómo considera el acceso a la empresa INRUE, C.A., para comprar sus productos

\begin{tabular}{ccc}
\hline Opciones & Frecuencia & Porcentajes \\
\hline Cerca & 3 & $14 \%$ \\
Muy cerca & 0 & $0 \%$ \\
Lejos & 11 & $50 \%$ \\
Muy lejos & 8 & $36 \%$ \\
Total & 22 & $100 \%$ \\
\hline
\end{tabular}

Fuentes: Aguilar, R., Alvarado, J. (2019)

En este punto se puede visualizar que existe un cierto nivel poblacional que no les es fácil acceder a la empresa y motivo por el cual se le deben dar mayores comodidades y facilidades de información y productos a los clientes tanto actuales como a los futuros clientes.

Cuadro $\mathbf{N}^{\circ}$ 12. Le gustaría que la empresa INRUE, C.A., tuviera un establecimiento de venta más céntrico

\begin{tabular}{ccc}
\hline Opciones & Frecuencia & Porcentajes \\
\hline Si & 22 & $100 \%$ \\
No & 0 & $0 \%$ \\
Total & 22 & $100 \%$ \\
\hline
\end{tabular}

Fuentes: Aguilar, R., Alvarado, J. (2019)

En vista de esta situación se hace notar claramente que una necesidad de tener un mejor acceso a los productos de la empresa así como de la información sobre los productos para ser adquiridos a través de una herramienta digital para cubrir las necesidades de los clientes. 
Cuadro $\mathbf{N}^{\circ}$ 13. En qué lugar preferiría la ubicación de dicho establecimiento

\begin{tabular}{ccc}
\hline Opciones & Frecuencia & Porcentajes \\
\hline Tocuyito & 1 & $4 \%$ \\
Valencia & 11 & $50 \%$ \\
Centro de la ciudad & 7 & $32 \%$ \\
Otros & 3 & $14 \%$ \\
Total & 22 & $100 \%$ \\
\hline
\end{tabular}

Fuentes: Aguilar, R., Alvarado, J. (2019)

Ante esta problemática se evidencia claramente que la mayoría de los clientes encuestados sienten la necesidad de tener el establecimiento dentro de la zona de Valencia para que se les haga más accesible para la compra de sus productos.

Cuadro $\mathbf{N}^{\circ}$ 14. Le gustaría contar con un asesor de ventas especializado para mayor comodidad para los clientes de la empresa INRUE, C.A.

\begin{tabular}{ccc}
\hline Opciones & Frecuencia & Porcentajes \\
\hline Si & 20 & $91 \%$ \\
No & 2 & $9 \%$ \\
Total & 22 & $100 \%$ \\
\hline
\end{tabular}

Fuentes: Aguilar, R., Alvarado, J. (2019)

Evidenciándose con esto que si existe la necesidad de mantener un canal de conexión para los clientes que sean asesorados en sus compras para acondicionar los productos a sus necesidades y con ello incrementar las ventas y un mejor posicionamiento dentro del mercado.

Cuadro $\mathbf{N}^{\circ}$ 15. Qué medio le gustaría para valorar los productos de INRUE, C.A.

\begin{tabular}{ccc}
\hline Opciones & Frecuencia & Porcentajes \\
\hline Prensa & 5 & $23 \%$ \\
Radio o TV & 8 & $36 \%$ \\
Instagram & 9 & $41 \%$ \\
Otros & 0 & $0 \%$ \\
Total & 22 & $100 \%$ \\
\hline
\end{tabular}

Fuentes: Aguilar, R., Alvarado, J. (2019) 
Con ello se visualiza la falta de una herramienta publicitaria para que los clientes logren valorar los productos y en el mercado actual se tipifica sobre las redes sociales y para nuestro proyecto sobre el marketing digital 4.0 .

Cuadro $\mathbf{N}^{\circ}$ 16. Recomendaría con sus proveedores y amigo/a los productos de la empresa INRUE, C.A.

\begin{tabular}{ccc}
\hline Opciones & Frecuencia & Porcentajes \\
\hline Si & 20 & $91 \%$ \\
No & 2 & $9 \%$ \\
Total & 22 & $100 \%$ \\
\hline
\end{tabular}

Fuentes: Aguilar, R., Alvarado, J. (2019)

En este punto se puede observar que son productos de muy buena calidad capaz de ser recomendados por sus clientes a sus proveedores y otros posibles clientes.

Cuadro $\mathbf{N}^{\circ}$ 17. Qué tipo de mejoras cree usted que serían favorables para impulsar al posicionamiento de la empresa INRUE, C.A.

\begin{tabular}{ccc}
\hline Opciones & Frecuencia & Porcentajes \\
\hline Mayor Publicidad & 8 & $34 \%$ \\
Mayor Ubicación & 6 & $30 \%$ \\
Mas variedad & 2 & $6 \%$ \\
Tiendas & 6 & $30 \%$ \\
Total & 22 & $100 \%$ \\
\hline
\end{tabular}

Fuentes: Aguilar, R., Alvarado, J. (2019)

Evidenciándose con esto que se debe promocionar más los productos de la empresa para aumentar la clientela y si la demanda sigue en crecimiento se abrirán otras tiendas para la comodidad y acceso para los clientes. 
Plan estratégico basado en el marketing digital 4.0 para fortalecer el posicionamiento en el mercado manufacturero caso estudio: INRUE

\section{C.A. Valencia, Estado Carabobo}

Con base en el diagnóstico realizado, se propone un plan estratégico basado en el marketing 4.0 con el fin de fortalecer el mercado manufacturero de la empresa INRUE C.A ubicada en Valencia, estado Carabobo, Venezuela, bajo los siguientes términos.

\section{Objetivo general del plan de marketing digital}

Establecer los requerimientos necesarios para la creación de planes estratégicos de marketing digital, tomando en cuenta el estudio de mercado realizado a los clientes del Estado Carabobo

\section{Objetivos específicos del plan de marketing digital}

Definir las estrategias de marketing, ventas y finanzas para lograr el éxito de la marca.

$\checkmark$ Impulsar las ventas de la empresa INRUE C.A mediante una campaña publicitaria en redes sociales.

$\checkmark$ Incrementar las ventas por menudeo mediante la creación de una tienda física en la ciudad de Valencia.

$\checkmark$ Inducir las ventas de los productos mediante una política de promoción de precios, con la finalidad de abarcar el mercado estadal.

\section{Marketing digital}

\section{Publicidad.}

INRUE C.A mediante la aplicación de una estrategia de crecimiento y posicionamiento de la marca con recursos tecnológicos y económicos incursionará con éxito en el mercado digital. Estas estrategias planteadas son las siguientes:

Publicar el lanzamiento de los servicios de fabricación y venta de ruedas industriales aplicando un bombardeo en redes sociales y medios de comunicación tradicional. Este método puede ser implementado por INRUE C.A, debido a que sus competidores no poseen presencia en el mercado digital. Luego lo deberán hacer mediante publicidad y promociones, a lo que INRUE C.A debe estar preparada y enfrentar a la competencia con las ventajas y relación costo - calidad.

Introducción Múltiple: Consiste en que INRUE C.A dé a conocer sus productos, mediante la publicidad en Internet y medios de comunicación tradicional como radio, prensa local y otros medios.

Facebook: A través de la creación y manejo de esta red social podrá publicar sin incurrir en gastos sus promociones $y$ productos. Las personas lo utilizan para mantenerse en contacto y actualizados con sus familiares y amigos, actualmente posee más de 12.000 .000 millones de usuarios es por ello que su publicidad puede ser efectiva y mediante sus herramientas se podrá segmentar al público a quien va dirigida la publicidad. 
Instagram: Mediante la creación y manejo de esta importante red social donde se encuentran aproximadamente 25.000 .000 de usuarios a nivel mundial interactuando a diario y visualizando publicidad se podrá tener un impacto mayor ya que mediante esta red social que está enfocada en fotografías se logrará dar a conocer sus productos e instalaciones de la empresa, así como la ubicación de la nueva tienda en Carabobo permitiendo así la interacción en tiempo real con los clientes.

Twitter: A través de la creación y manejo de esta red social que posee a nivel mundial más de 20.000.000 de usuarios se podrá mediante la emisión de 140 caracteres promocionar la marca y publicitar sus productos y nueva ubicación de la tienda.

Página Web: Mediante la creación de la Página Web se logrará promocionar y publicitar los diversos productos y servicios e información de la empresa, donde INRUE C.A podrá tener banners informativos de sus promociones y descuentos así como la ubicación de su nueva tienda en la región carabobeña e interacción directa con sus clientes.

\section{Promoción de Productos Online}

Es importante tener en cuenta que este tipo de promoción online es una herramienta poderosa y novedosa para transmitir confianza y atraer más clientes a la empresa.

Ventas: El departamento de ventas en conjunto con el departamento de crédito y cobranzas, tendrá la responsabilidad de las ventas realizadas mediante Internet, deben facilitar a los clientes los números de cuentas bancarios para facilitar el pago de los servicios prestados por la organización. De igual forma, deben dar cumplimiento a los requerimientos necesarios para promocionar a los clientes el pago online de los productos mediante tarjetas de crédito, débito o transferencias bancarias, así como también la captación de los datos necesarios para la facturación y envió de ser necesario de los productos.

\section{Estrategia de Marketing}

Crecimiento del mercado manufacturero de INRUE C.A: Consiste en crecer en el mercado de referencia en el cual se maneja la organización. Se utiliza cuando no se ha explorado completamente las oportunidades ofrecidas por los productos que dispone y los mercados que cubre, y esto es lo que caracteriza a esta empresa, se puede aplicar la estrategia de penetración del mercado, a través del uso de componentes como el desarrollo de la demanda que en este caso logrará el aumento de la tasa de ocupación y esto consiste en atraer nuevos clientes para así incrementar sus ventas. Esto no requiere de un gran presupuesto solo debe estar dirigido a los diversos canales de comunicación masiva en ventas para obtener una mayor efectividad y rentabilidad.

Por otra parte, INRUE C.A podrá aumentar la tasa de penetración en el mercado con la implementación de acciones orientadas a la obtención de un mayor número de compras de los productos que 
ofrece en el mercado y mayor frecuencia de clientes actuales, enfocados en el marketing digital.

\section{Diseño de la Página Web Informativa}

En este recurso se deberá ofrecer información sobre la organización, su misión, visión, objetivos, productos, ubicación, datos de contacto, redes, posibilidades de chat, entre muchos otros aspectos que faciliten la interacción de los posibles clientes con la empresa, generando un clima de seriedad y confianza.

\section{Presencia en Redes Sociales}

Se trata de generar y alimentar con alta frecuencia las redes sociales institucionales de la empresa, tales como Facebook, Twitter, Instagram, con las cuales las personas están familiarizados y las usan con una alta frecuencia.

\section{Posicionar la marca en el Estado Carabobo}

Posicionamiento de la Página Web y Redes Sociales en los buscadores. Para que INRUE C.A logre posicionar su página web y redes sociales en Internet, deberá realizar las siguientes recomendaciones:

- Destacar la calidad de los productos comercializados y los beneficios que proporcionan al cliente.

- Definir un slogan clave a posicionar, el cuál debe estar directamente relacionado con la temática que se manejará en la página web y redes sociales, como "Servicio de calidad a un bajo costo".

- Alojar la página web en los buscadores que poseen más tráfico a los sitios web, como lo son: Google, Msn y Yahoo con la finalidad de que reciba mayor cantidad de visitas en el portal web y redes sociales.

\section{CONCLUSIONES}

Durante el desarrollo de la investigación, se observó como las empresas luchan constantemente por sobresalir con productos y tecnologías innovadoras que le garanticen la presencia en el mercado, sea cual fuere el segmento en el que se encuentran o en el cual desean posicionarse. Así como también, se observó a la importancia que debería existir en la relación entre el equipo de planeación estratégica de marketing y el resto de las personas involucradas, con el único objetivo de alcanzar el éxito de la nueva planificación.

La empresas en muchas ocasiones incursionan en las redes sociales como mecanismo de marketing con muy poco éxito, de allí la importancia de que estas medidas se fundamenten en un estudio sistematizado, como el realizado en esta investigación, en el cual partiendo de los análisis internos y externos, a través de las entrevistas, los cuestionarios y la aplicación de la matriz DOFA, se arrojan elementos valiosos y certeros para planificar con un mínimo margen de error en estas necesarias estrategias de marketing, en un mercado global que cada vez es más cambiante, 
dinámico y competitivo, con unos clientes que son más exigentes y buscan las mayores facilidades para adquirir bienes y servicios.

Estamos al pie de una nueva era, la digital, y dependerá de la capacidad de adaptación de las empresas su permanencia en el tiempo. De allí que esta investigación y sus resultados sea de relevancia, por su capacidad de extrapolar su aporte a otras empresas con similares condiciones.

REFERENCIAS

Barrera, E. (2008). Mercadeo. Medellín: COLCIENCIAS
Castilla (2015). Concepto y Origen de la palabra estrategia. Disponible en: http://neuroempresarial.blogspot.com/20 11/05/concepto-y-origen-de-lapalabra.html. Consulta: 2019, Junio 15 Cobra, Maurice (2007). Administración del Marketing. Sao Paulo. Atlas

Concha, M. (2008). Microempresas y microempresarios: una visión total. Caracas: Vadell Hnos

Kerin, C., Lambin, J. (2004). Marketing Estratégico. McGraw-Hill. Madrid

Luther, W. (2008). El Plan de Mercadeo. (3 ed.). Editorial Norma. Madrid 